\title{
COGNICIÓN Y RETÓRICA: \\ BASES BIOLÓGICAS DEL SIGNIFICADO \\ Y LA COMPRENSIÓN ${ }^{1}$
}

\author{
FRANCISCO ARENAS-DOLZ \\ Universitat de València
}

\begin{abstract}
RESUMEN: El propósito de este trabajo es doble: por un lado, explorar aquellos enfoques que entienden los procesos cognitivos como corporizados y determinados por nuestra interacción con el medio, y no meramente como un sistema de tratamiento de la información; y, por otro lado, mostrar su conexión con los mecanismos neurofisiológicos que subyacen al significado y a la comprensión, presentando un panorama general a partir de algunos estudios recientes en neurorretórica.
\end{abstract}

PALABRAS CLAVE: plasticidad cerebral, percepción intermodal, neuronas espejo, neurorretórica, orígenes del lenguaje, sinestesia.

\section{Cognition and Rhetoric: Biological Basis of Meaning and Understanding}

ABSTRACT: The aim of this paper is twofold: on the one hand, to explore those approaches that understand cognitive processes as embodied and as a result of our interaction with the environment, and not merely as as a information processing system; and, on the other hand, to show their connexion with the neurophysiological mechanisms underlying meaning and understanding, giving and overview of some of the insights from recent studies in neurorhetorics.

KEY WORDS: brain plasticity, cross-modal perception, mirror-neurons, neurorhetorics, origins of language, synesthesia.

\section{INTRODUCCIÓN}

La revista estadounidense Rhetoric Society Quarterly dedicó en 2010 un volumen monográfico a definir y explorar las características más importantes de la neurorretórica, entendiendo con este término una orientación de la comunidad científica que estudia las dinámicas de la comunicación y la cognición a la luz de los resultados obtenidos desde los años noventa del siglo pasado gracias a nuevas técnicas de neuroimagen (como la EMT, estimulación magnética transcraneana, o la PET, tomografía por emisión de positrones), capaces de fotografiar con enorme precisión la actividad de nuestro cerebro mientras observamos distintas situaciones, deliberamos acerca de cómo realizar una acción determinada o reelaboramos las percepciones corporales. La neurorretórica constituiría tanto un ámbito de estudios interdisciplinarios —que aúna retórica clásica, lingüística, teoría de la literatura, neurofisiología, cognitivismo y

1 Este trabajo se enmarca en el proyecto «Juicio moral, justicia y democracia en perspectiva neuroética» (FFI2013-47136-C2-1-P) financiado con fondos del MINECO. 
psicología experimental — como aquella actividad mediante la cual manipulamos las operaciones neuronales de nuestro auditorio y lo convencemos de que haga o crea algo. La neurorretórica es pues tanto un objeto de estudio como al mismo tiempo el estudio de ese objeto².

Jordynn Jack, editora del monográfico, propone que nos imaginemos a nosotros mismos mientras miramos en televisión un documental dedicado a una figura histórica como Martin Luther King. El presentador es un nuevo tipo de experto, un neuro-orador, el doctor Cráneo. Mediante escáneres neuronales y una cartografía de las sinapsis, el neuro-orador sabe que la célebre frase «I have a dream» activa en el cerebro el núcleo accumbens, de modo que las células dopaminérgicas, que participan de importantes procesos biológicos como el movimiento, la motivación y la función intelectual, se relajan inmediatamente, provocando una sensación de placer difuso, lo que conduce al auditorio a conectar empáticamente con el discurso del líder pacifista estadounidense. El doctor Cráneo no procede por intuición, ni tiene un talento especial como comunicador, pero dispone de datos científicos que le dicen qué hacer para ser persuasivo. De acuerdo con Jack, la neurorretórica consistiría tanto en la actividad manipuladora del doctor Cráneo con instrumentos inconcebibles hace apenas veinte años como en el estudio de estos instrumentos y de las reacciones suscitadas en el auditorio.

El efecto de la manipulación es tanto mayor cuanto más escuchemos las palabras del doctor Cráneo. Como ha demostrado Vittorio Gallese, estudioso de las neuronas espejo, nos «encarnamos» en el emitente, sentimos lo que él siente, simulamos neuronalmente lo que él dice y los gestos que hace al decirlo ${ }^{3}$. To embody, 'encarnar' o 'corporizar', es uno de los verbos más repetidos por las neurociencias. De hecho, según Jack, cuando el documental se interrumpe por una pausa publicitaria vemos a nuestro doctor Cráneo promocionando productos y, mientras activa neuronalmente la implicación empática del auditorio, le promete devolverle el dinero si no queda satisfecho. Podría parecer un escenario de ciencia ficción, pero forma parte de nuestra realidad cotidiana.

Las ciencias cognitivas han dado un nuevo impulso a la retórica. Si el renacimiento de la retórica a mediados del siglo xx fue promovido por la exigencia de enfocar el estudio de la realidad desde una perspectiva argumentativa, basada en la razonabilidad y la deliberación, necesaria para orientar la convivencia democrática en sociedades pluralistas, en la actualidad la revitalización de la retórica está asociada a los avances en las ciencias cognitivas y en particular a las distintas interpretaciones que se han dado sobre el carácter situado de la

2 Jack, J. y Appelbaum, L. G., "This is your brain on rhetoric': Research directions for neurorhetorics», en: Rhetoric Society Quarterly, 40/5, 2010, 419.

3 Gallese, V.; Migone, P. y Eagle, M. N., «La simulazione incarnata: i neuroni specchio, le basi neurofisiologiche dell'intersoggettività ed alcune implicazioni per la psicoanalisi», en: Psicoterapia e Scienze Umane, 12.3, 2006, 543-548; Gallese, V., "Mirror neurons and the social nature of language: The neural exploitation hypothesis», en: Social Neuroscience, 3/3-4, 2008, 317-333. 
cognición (corporizada, enactiva, distribuida, incrustada, extendida, situada). Desde una perspectiva interdisciplinaria, la convergencia de las ciencias cognitivas y la retórica ha propiciado el desarrollo de un novedoso ámbito de estudios, la neurorretórica, que explora tanto las funciones cerebrales relacionadas con la persuasión y la argumentación como la manera en que los hallazgos de la investigación neurocientífica se presentan retóricamente.

Como argumentaré, la discusión entre retórica y ciencia cognitiva —propiciada desde enfoques pragmatistas, fenomenológicos o constructivistas, entre otros- podría ofrecer intuiciones fructíferas tanto a retóricos como a teóricos cognitivos. Los descubrimientos neurocientíficos nos han dado la oportunidad de pensar la retórica no como arte de la persuasión, teoría de la argumentación o teoría literaria, sino fundamentalmente como ciencia acerca de cómo estructuramos nuestro conocimiento a través del lenguaje. Además, las ciencias cognitivas han favorecido el estudio de una retórica no desvinculada del cuerpo humano o de los aspectos situacionales, sino como parte de ellos. El cuerpo es un vehículo que permite la expresión de las subjetividades, nos dota de conocimiento práctico y contribuye a la creación de un espacio social.

\section{De LA COGNICIÓN CORPORIZADA A LA MENTE EXTENDIDA}

El paradigma clásico de las ciencias cognitivas consideraba la mente como un sistema computacional de procesamiento de la información ${ }^{4}$. Las neurociencias han contribuido a revisar esta concepción de la mente y los conceptos tradicionales sobre los procesos cognitivos. Poco a poco se han ido desarrollando dentro de la ciencia cognitiva otras perspectivas que entienden la cognición en un ambiente ${ }^{5}$. En este marco se inscriben enfoques alternativos tales como la cognición corporizada, enactiva, distribuida, incrustada, extendida o situada. Aunque hay varias diferencias entre estas perspectivas, todas ellas rechazan las ideas más tradicionales de la ciencia cognitiva basadas en lo mental como un suceso privado, racional, abstracto y desencarnado, posibilitado por mecanismos rígidos de tipo computacional que equiparan a los seres vivos con máquinas y relegan el papel activo de los organismos en la constitución de su experiencia a un papel secundario. Y comparten una serie de características centrales para comprender el sistema cognitivo, como la interacción y el dinamismo: la interacción dinámica enmarcada en un cuerpo y la comprensión simultánea de factores corporales, neurales, emocionales y ambientales

\footnotetext{
4 Newell, A. y Simon, H. A., "Computer science as empirical inquiry», en: Communications of the ACM, 19, 1976, 113-126.

5 Rumelhart, D. E.; McClelland, J. L. y PDP Research Group (eds.), Parallel distributed processing, MIT Press, Cambridge 1986; VAN GELDER, T., "What might cognition be, if not computation?», en: Journal of Philosophy, 91, 1995, 345-381; Id., «The dynamical hypothesis in cognitive science», en: Behavioral and Brain Sciences, 21, 1998, 615-628.
} 
que interactúan ${ }^{6}$. El punto de partida de los defensores de estas perspectivas ambientalistas es el hecho de que hay formas de estar en el mundo que no implican representaciones neutrales respecto a la acción, esgrimiendo principios metodológicos de carácter científico para postular una concepción de nuestra mente más acorde con nuestras historia evolutiva y nuestra inserción radical en el entorno material y social.

1. La tesis de la cognición corporizada afirma que la actividad cognitiva es asunto del organismo entero, no sólo de su cerebro. El primer anclaje en el mundo para los seres humanos - y otras especies- es la interacción del cuerpo con el entorno. La situacionalidad parece arraigar en la corporalidad que acompaña a todo proceso cognitivo. Los mecanismos básicos de la comprensión humana dependen de estructuras preconceptuales y no-proposicionales organizadas imaginativamente a partir de nuestra experiencia corporal. Entender la cognición como corporizada implica entender lo mental como un conjunto de fenómenos públicos, distribuidos y dinámicos, debido a la naturaleza comunicativa del cuerpo. Esta teoría atiende en particular al papel de los afectos ${ }^{7}$ y se ha convertido en un elemento imprescindible en buena parte de los estudios sobre el lenguaje, especialmente en relación con la teoría de la metáfora conceptual ${ }^{8}$.

2. La tesis de la cognición situada enfatiza el lugar central del cuerpo, su motricidad y experiencia, así como el contexto ambiental en los procesos cognitivos. Los investigadores que pusieron en marcha esta teoría insistieron en que, ante la complejidad cambiante de las situaciones, resultaba imprescindible reintroducir el entorno de la actividad, ausente de la versión tradicional del cognitivismo. Así, por ejemplo, Lucy Suchmanª empleó esta tesis en apoyo de su teoría de la planificación, recusando la idea de procesos de planificación vistos como determinantes de la acción: según ella, es más bien la acción situada la que genera los planes. No es que los planes no tengan importancia en los actos cotidianos; más bien al contrario: ayudan a los sujetos a dar sentido a la acción.

Al igual que la tesis de la cognición corporizada, la tesis de la cognición situada considera la emoción como una forma de vincularse hábilmente con el mundo, y de esta interacción emerge lo significativo para el sujeto que lo experimenta ${ }^{10}$. La experiencia emocional no es únicamente una

6 BRоокs, R. A., «Intelligence without representation», en: Artificial Intelligence, 47, 1991, 139-159.

7 Damasio, A. R., El error de Descartes. La emoción, la razón y el cerebro humano, Crítica, Barcelona 2001.

8 Lakoff, G. y Johnson, M., Metáforas de la vida cotidiana, Cátedra, Madrid 2007.

9 Suchman, L., Plans and situated actions: The problem of human-machine communication, Cambridge University Press, New York 1987; Ead., «Representing practice in cognitive sciences», en: Human Studies, 11/2-3, 1988, 305-325.

10 Griffiths, P. E. y Scarantino, A., «Emotions in the wild», en: Robbins, Philip y Aydede, Murat (eds.), The Cambridge handbook of situated cognition, Cambridge University Press, 
cuestión subjetiva, sino que las emociones son cualidades que surgen en la interacción entre el organismo y el ambiente que conoce; además, son interacciones experimentadas y compartidas con otras personas, que se van aprendiendo a lo largo de toda la vida.

3. La tesis de la cognición enactiva, originalmente planteada por el psicólogo estadounidense Jerome S. Bruner a finales de los años 60 del siglo xx, fue desarrollada por el neurobiólogo y filósofo chileno Francisco J. Varela. Esta teoría diverge de otros enfoques ambientalistas, al subrayar que, además de la importancia del cuerpo y la acción, para la emergencia de una actividad cognitiva es necesario que esa actividad tenga un soporte físico con unas propiedades organizativas especiales que emergen en los organismos, los cuales en cuanto cuerpos biológicos - autónomos o autopoiéticos-se regulan y generan experiencias de sentido ${ }^{11}$. Así, el conocimiento no puede reducirse a la recepción pasiva de información, sino que se construye durante el desarrollo de los organismos, que enactúan sus mundos significativos plenos de sentido. El conocer no es solamente un fenómeno vivo, sino que es vivido, pues siempre una experiencia de conocimiento tiene como fundamento la vivencia en primera persona. La cognición dependerá del carácter corporizado de las aptitudes sensomotoras que, a su vez, están inmersas en un contexto biológico, psicológico y cultural más amplio. El mundo no existe independientemente de la forma en que el sistema lo hace emerger mediante sus acciones en él ${ }^{12}$.

4. La tesis de la cognición distribuida, desarrollada a mediados de 1980 por Edward Hutchins, incide en los aspectos sociales de la cognición. Desde este marco se entiende la cognición como el proceso de la información que se produce a partir de la interacción con símbolos en el mundo. El conocimiento humano y la cognición no están confinados en el individuo, sino también en espacios de memoria distribuidos, en hechos, o en el conocimiento de los objetos de nuestro entorno. Hutchins propone que la cognición y el conocimiento no se limitan a un individuo, sino que se distribuyen a través de objetos, personas, objetos y herramientas en el entorno ${ }^{13}$.

Se ha concebido la cognición humana como si fuese algo poseído y residiese en la cabeza de los individuos sin prestar atención, en general, al estudiarla, a los entornos social, físico y artificial donde se produce. Frente a ello, Gavriel Salomon ha propuesto un tratamiento basado en la idea de una cognición distribuida entre los individuos y un conocimiento

Cambridge 2009; Mesouita, B., «Emoting: A contextualized process», en: Mesouita, B.; BaRRetT, L. F. y Sмith, E. R. (eds.), The mind in context, Guilford Press, New York 2010, 83-104.

11 Varela, F. J.; Thompson, E. y Rosch, E., De cuerpo presente. Las ciencias cognitivas y la experiencia humana, Gedisa, Barcelona 1992.

12 Maturana, H., Erkennen: Die Organisation und Verkörperung von Wirklichkeit, Friedrich Vieweg und Sohn, Braunschweig/Wiesbaden 1982; Maturana, H. y Varela, F. J., El árbol del conocimiento. Las bases biológicas del entendimiento humano, Debate, Madrid 1990.

13 Hutchins, E., Cognition in the wild, MIT Press, Cambridge 1995. 
construido socialmente a través de esfuerzos cooperativos dirigidos a alcanzar objetivos comunes y desarrollados en entornos culturales, donde la información es objeto de un procesamiento que tiene lugar entre los individuos y las herramientas y artefactos que proporciona la cultura ${ }^{14}$.

Algunos autores han sugerido la existencia de una cognición colectiva, lo que nos permitiría aprovecharnos de los artefactos diseñados por otros, compartiendo las buenas ideas a través del tiempo. Por un lado, Pierre Lévy ha acuñado el concepto de inteligencia colectiva para referirse a la producción, distribución y circulación del conocimiento en el actual mundo de los avances de la tecnología de la información y comunicación ${ }^{15}$. Por otro lado, George Siemens y Stephen Downes han desarrollado la teoría del conectismo o conectivismo ${ }^{16}$. Si antes la organización del conocimiento era clara y estática en jerarquías y contenedores, hoy esa organización consiste en redes dinámicas y ecologías — modelos sensibles a la adaptación, que se ajustan y reaccionan a los cambios. Con base en las neurociencias, el cognitivismo, la teoría de redes, la teoría del caos, los sistemas adaptativos complejos y disciplinas afines, el conectivismo concibe el conocimiento ya no como una actividad individual sino como un proceso basado en conexiones, que depende de los individuos, pero reside en el colectivo ${ }^{17}$.

5. La tesis de la cognición incrustada sostiene que el cerebro es un órgano relacional incrustado en las interacciones significativas de un ser vivo con su entorno. Como órgano inscrito en nuestra naturaleza corporal, y en consecuencia en nuestra conciencia e intencionalidad, el cerebro soporta las relaciones con el mundo de manera dinámica, no reproduciendo copias fieles de una realidad preestablecida, sino operando como un sistema complejo que crea sus propios significados, los cuales se encarnan en forma de patrones de actividad neuronal. Como parte de un sistema dinámico, la función neuronal no puede verse desligada de su incrustación en contexto, lo que rechazaría la consideración del cerebro como abstrayendo información exterior y procesándola a través de módulos especializados. Toda cognición se incrusta en situaciones en las cuales el agente ha de responder

14 Salomon, G. (ed.), Cogniciones distribuidas. Consideraciones psicológicas y educativas, Amorrortu, Buenos Aires 2001.

15 Lévy, P., Inteligencia colectiva. Por una antropología del ciberespacio, Organización Panamericana de la Salud, Washington 2004.

16 Siemens, G., Conociendo el conocimiento [en línea], Nodos Ele, Madrid 2010 [consultado 30 mayo 2016]. Disponible en Internet: <http://www.nodosele.com/editorial>; Downes, Stephen, «An introduction to connective knowledge», en: Hug, T. (ed.), Media, knowledge \& education: Exploring new spaces, relations and dynamics in digital media ecologies [en línea], Innsbruck University Press, Innsbruck 2008, 77-102 [consultado 30 mayo 2016]. Disponible en Internet: <http://www.oapen.org/search?identifier=449459>.

17 BarabásI, A.-L., Linked: How everything is connected to everything else and what it means for business, science, and everyday life, Perseus Publishing, Cambridge 2002; CHRISTAKIS, N. A. y Fowler, J. H., Conectados. El sorprendente poder de las redes sociales y cómo nos afectan, Taurus, Madrid 2010. 
a las novedades imprevistas del ambiente. Para Andy Clark, por ejemplo, la interacción cuerpo-mundo es constitutiva de la mente y nos presenta la idea de una mente esencialmente corporizada la cual, además, se encuentra encajada o incrustada en el mundo en el que actúa ${ }^{18}$.

6. La tesis de la cognición extendida sostiene que ciertos procesos cognitivos deben entenderse como situados, corporizados y orientados hacia el logro de objetivos concretos. Estos procesos se suelen desarrollar en situaciones de la vida real en interacción con el ambiente material y social. En estas situaciones, el cerebro, el cuerpo y el mundo se llegan a coordinar de tal manera que hacen que la mente se extienda hacia el mundo exterior. Andy Clark y David Chalmers sostienen que mientras que algunos estados mentales y experiencias pueden definirse internamente, existen muchos otros en los que los procesos de atribución de significado incluyen algunos componentes localizados fuera del cráneo ${ }^{19}$. Estos elementos externos que forman parte del ambiente social y material son parte de los sistemas cognitivos responsables de tales procesos. En consecuencia, algunos procesos cognitivos no deben seguir entendiéndose como constreñidos por los límites físicos del cerebro, sino que emergen, se desarrollan y se extienden a lo largo de redes interactivas que integran y sincronizan funcional y estratégicamente el cerebro, el cuerpo y el mundo físico y social.

Clark describe las circunstancias en que los artefactos culturales -incluyendo el lenguaje - y los avances tecnológicos pueden tener una vida cognitiva al convertirse en una extensión de la mente humana ${ }^{20}$. Clark sostiene que ciertos artefactos culturales y dispositivos tecnológicos trascienden su funcionalidad básica como herramientas que simplemente aumentan las capacidades humanas. Así, la coordinación entre el cerebro, el cuerpo y el mundo facilita la construcción de nuevos sistemas cognitivos extendidos que guían procesos cognitivos orientados a la resolución de problemas en situaciones reales.

Si la tesis de la cognición situada insistía en el papel del entorno como esencial en los procesos cognitivos, la teoría de la mente extendida no es sino un modo paradigmático de la cognición situada. La mente misma se extiende más allá de los estados internos al organismo. Los aspectos externos son constituyentes del sistema, componentes de los procesos cognitivos. Aunque sea imposible construir un sistema cognitivo compuesto exclusivamente de elementos externos, no son menos esenciales que los componentes internos.

A partir de estas concepciones ambientalistas de la mente, que se alejan del paradigma cognitivista clásico, las neurociencias han estudiado las

18 CLARK, A., Being there: Putting brain, body, and world together, MIT Press, Cambridge 1997.

19 Clark, A. y Chalmers, D. J., «The extended mind», en: Analysis, 58, 1998, 7-19.

20 CLARK, A., Supersizing the mind: Embodiment, action, and cognitive extension, Oxford University Press, New York 2008. 
bases neuronales que permitan justificar este cambio teórico. En este contexto, como veremos a continuación, ocupa un lugar destacado la investigación neurorretórica.

\section{Persuasión, ARgumentación y NEURORRETÓRICA}

1. Las ciencias cognitivas han dado un nuevo impulso a la retórica, que ya Aristóteles había definido como la facultad de descubrir lo que es adecuado en cada caso para persuadir, revalorizándola frente a Platón, quien en el Gorgias la había desacreditado, confiándole la tarea de recurrir a argumentos persuasivos, es decir, no de tipo puramente racional, como los formulados por la lógica demostrativa y la dialéctica ${ }^{21}$. Antes de que las neurociencias cognitivas le abriesen nuevos horizontes, la retórica tuvo un renacimiento gracias a Chaïm Perelman y Lucie Olbrechts-Tyteca, quienes en 1958 publicaron el célebre Tratado de la argumentación, oponiéndose a una idea restrictiva y cartesiana de razón, entendida como cálculo, modelo matemático y procedimiento formal ${ }^{22}$. A juicio de los autores, la retórica nos enseñaría a entender la razón como un proceso, una negociación de puntos de vista diferentes, un diálogo en el que emerge la razonabilidad y la verdad compartida. Perelman y Olbrechts-Tyteca convierten a la retórica en el campo de experimentación de la argumentación - diferente de la demostración, utilizada en las ciencias exactas-, un modo de enfocar el estudio de la realidad necesario para la convivencia social. De aquí se desprende que la retórica es propiamente una teoría de la acción humana, el organon de la deliberación acerca de lo bueno y lo conveniente ${ }^{23}$.

Así, desde los años sesenta, la retórica se ha presentado como una defensora de la razonabilidad y la deliberación, atenta al auditorio, y su importancia ha sido notable en el mundo de la comunicación política y de la publicidad. Las investigaciones interdisciplinares de los psicólogos cognitivos, y otros estudiosos provenientes de ámbitos diversos, unidas a las revolucionarias técnicas de neuroimagen desarrolladas en los años noventa, han apelado a la necesidad de revitalizar la retórica para afrontar

21 Aristóteles, Retórica, 1355b, Gredos, Madrid 1992; Platón, Gorgias, 465d, Gredos, Madrid 1992.

22 Perelman, C. y Olbrechts-Tyteca, L., Tratado de la argumentación. La nueva retórica, Gredos, Madrid 1989.

23 Bеuснот, M., La retórica como pragmática y hermenéutica, Anthropos, Barcelona 1998; Coronel Ramos, M. A. y Giménez Moreno, R., «Cognición y retórica», en: Arbor, 177.697, 2004, 41-58; Conill, Jesús, "Retórica y vida humana», en: Arenas-Dolz, F. (ed.), Retórica y democracia. Perspectivas críticas sobre el estado de la investigación, Institució Alfons el Magnànim, Valencia 2012, 323-339; Ramírez, J. L., "Seguidores o continuadores de la retórica clásica», en: Arenas-Dolz, Francisco (ed.), o.c., 341-366. 
con medios renovados, desde la centralidad de los procesos mentales, el estudio del conocer humano.

2. Para el nacimiento de la neurorretórica ha sido fundamental la distinción entre esquema (schema) y guión (script). La teoría del esquema fue formulada por primera vez en los años veinte del siglo pasado en el ámbito de la psicología de la Gestalt, y experimentó un auge considerable con posterioridad gracias a las investigaciones sobre inteligencia artificial desarrolladas por Marvin Minsky ${ }^{24}$, Roger Schank y Robert Paul Abelson ${ }^{25}$. La teoría del esquema - también conocida como teoría del marco o del encuadre (frame) - considera que cada nueva experiencia se comprende a partir de una confrontación con un modelo estereotípico derivado de experiencias similares registradas en la memoria: cada nueva experiencia se evalúa a partir de su adecuación o no con un esquema previo.

En consecuencia, el conocimiento puede entenderse como un conjunto de esquemas mentales interconectados, orientados a seleccionar, codificar y valorar los estímulos provenientes de la experiencia. Estos esquemas no sólo permiten la conceptualización y descodificación de las informaciones, sino también activan los procesos mentales de abstracción y categorización. Las experiencias se van sedimentando y pueden ampliarse y modificarse a medida que se experimentan nuevas vivencias identificadas semánticamente. El reconocimiento del contenido semántico de una situación dirige nuestra mente hacia la comprensión del contexto discursivo. Pero nuestro conocimiento no sólo cumple una función de conservación de los esquemas existentes (schema preserving), sino que también puede crearse un efecto de ruptura de esos esquemas (schema disruption), que lleva a los destinatarios a modificar sus estructuras cognitivas. Esta ruptura puede producirse sin desmantelar totalmente el esquema anterior, sino haciendo leves cambios, sustituyendo o integrando elementos que ya no son coherentes en el nuevo contexto discursivo (schema adding), pero también el proceso de ruptura de un esquema previo puede conducir a una transformación profunda (schema refreshment).

Sin embargo, la mera competencia para clasificar como esquemas situaciones que experimentamos a diario no es suficiente si queremos comprender la realidad. De hecho, el esquema se refiere sólo a objetos estáticos o relaciones; son necesarios también aquellos procesos dinámicos que los cognitivistas y los neurocientíficos llaman guiones (scripts). Un esquema proporciona el paradigma semántico de un evento, su significado, mientras que el guión articula sintácticamente aquello que sucede en los esquemas, es decir, el orden secuencial de los acontecimientos; sin el primero no se comprende nada, sin el segundo no ocurre nada. La adquisición de

24 Minsky, M. L., La sociedad de la mente. La inteligencia humana a la luz de la inteligencia artificial, Galápago, Buenos Aires 1986.

25 Schank, R. C. y Abelson, R. P., Guiones, planes, metas y entendimiento. Un estudio de las estructuras del conocimiento humano, Paidós, Barcelona 1987. 
esta doble competencia narrativa nos permite actuar y comprender las acciones de los otros. Schank y Abelson distinguen tres tipos de guiones: a) situacionales, referidos al horizonte de expectativas de situaciones cotidianas; b) personales, referidos a los roles de los individuos; c) instrumentales, referidos a las acciones necesarias para alcanzar un fin.

En síntesis, los esquemas cognitivos - fundamentales para reconocer el contexto útil para la comprensión tanto de la realidad fenoménica como de los mundos de la ficción narrativa- se estructuran en guiones. La memoria almacena las experiencias y los recuerdos personales más significativos y cataloga las vivencias, archivándolas en categorías generales, lo que permite anclar los datos de la experiencia humana en conocimientos ya almacenados en la memoria del individuo mediante esquemas, basados en la representación estática de los objetos y de sus características, y guiones, basados en la estructura dinámica de los acontecimientos.

3. La distinción entre esquema y guión nos permite comprender mejor cuáles son los procesos que conducen a la lectura de una palabra. Según Stanislas Dehaene, en un primer momento el ojo lee la palabra, la cual después se confronta con un archivo léxico, que cada uno de nosotros tiene en el cerebro $^{26}$. En el caso de no reconocer la palabra se activa la verificación: leemos de nuevo el término, tratamos de interpretarlo a través del contexto y de compararlo con palabras similares. La lectura de la palabra, sostiene Dehaene, comporta una operación de separación hasta llegar a la identificación de las distintas partes que la componen. Por su parte, para Maryanne Wolf no existe un área del cerebro predispuesta para la lectura, sino que la especie humana ha aprendido a leer gracias a la plasticidad cerebral utilizando capacidades que ya poseía ${ }^{27}$. Las operaciones de escritura y lectura han requerido un notable esfuerzo compensado con el surgimiento de nuevas conexiones entre los circuitos neuronales.

Lingüistas y antropólogos como Walter J. Ong ${ }^{28}$, Eric A. Havelock ${ }^{29}$ y Jack Goody ${ }^{30}$ se ocuparon de las relaciones entre oralidad y escritura y demostraron cómo éstas han modificado el conocer humano. Actualmente en el ámbito de las ciencias neurocognitivas un destacado número de investigadores sigue estudiando las transformaciones de la lectura y la escritura en el modo de pensar y en el incremento de las conexiones entre

26 Dehaene, S., Les neurones de la lecture, Odile, Paris 2007; Id., El cerebro lector, Siglo XXI, Buenos Aires 2014.

27 Wolf, M., Cómo aprendemos a leer. Historia y ciencia del cerebro y la lectura, Ediciones B, Barcelona 2008 .

${ }_{28}$ ONG, W. J., Oralidad y escritura. Tecnologías de la palabra, Fondo de Cultura Económica, México 1987.

29 Havelock, E. A., Prefacio a Platón, Visor, Madrid 1994.

30 Goody, J., La lógica de la escritura y la organización de la sociedad, Alianza, Madrid 1990. 
los circuitos neuronales. Por ejemplo, David Hermann ${ }^{31}$ y Lisa Zunshine ${ }^{32}$, siguiendo a Lev S. Vygotski ${ }^{33}$, Marvin Minsky ${ }^{34}$ y Jerome S. Bruner ${ }^{35}$, entre otros, se han centrado en la importancia del pensamiento narrativo como instrumento cognitivo que permite interpretar la realidad. Según Hermann, vivimos inmersos cotidianamente en universos narrativos que crean horizontes de expectativas. Para este autor, la narrativa es capaz de ofrecer modelos de comprensión conceptual de las situaciones y de cooperar en la construcción espacio-temporal del actuar humano. Es evidente que la narrativa, hecha de actores, eventos y ambientes diversos, nos aporta modelos de comprensión conceptual de las situaciones, construye horizontes de expectativas y nos ayuda a tomar decisiones para la acción.

4. La teoría del esquema y del guión ha sido empleada por la neurorretórica para explicar el fenómeno de la caracterización, entendido como los atributos peculiares de alguien o algo. La memoria semántica es por definición compartida socialmente: con el transcurso del tiempo los esquemas tienden a convertirse en estereotipos, generalizaciones inducidas por la colectividad, que influyen tanto en las percepciones como en los comportamientos de los individuos. Ya Aristóteles había tratado este asunto al elaborar las nociones de endoxa ${ }^{36}$-las opiniones comunes de una colectividad- y $\operatorname{topos}^{37}$ - la forma de decir o de pensar de una colectividad. Demostrando cómo cada individuo memoriza y almacena más fácilmente en la memoria informaciones vinculadas con expectativas formuladas sobre esquemas adquiridos, la neurorretórica explica no sólo la resistencia y la difusión extraordinaria de los estereotipos, sino también por qué el conocimiento esquemático se basa en modelos probabilísticos e ignora las excepciones, al menos tendencialmente. Más todavía, si las informaciones recibidas son coherentes con el esquema activado, se consolidan como estereotipos. La elección de un esquema es fruto de la consideración de una serie de factores, como la frecuencia con que aparece el esquema en varios contextos reales o ficticios, las metas que el destinatario se propone alcanzar o la situación en que se encuentra. Estos procesos de estereotipación no son estáticos, sino que son consecuencia de la interacción entre la percepción individual, el entorno y las construcciones y valores culturales. La

31 Herman, D., Story logic: Problems and possibilities of narrative, University of Nebraska Press, Lincoln 2002; Id. (ed.), Narrative theory and the cognitive sciences, Center for the Study of Language and Information, Stanford 2003.

32 Zunshine, L., Why we read fiction? Theory of mind and the novel, Ohio State University Press, Columbus 2006.

${ }_{33}$ Vygotski, L. S., El desarrollo de los procesos psicológicos superiores, Crítica, Barcelona 2000 .

MinSKY, M. L., o.c.

35 Bruner, J. S., La fábrica de historias. Derecho, literatura, vida, Fondo de Cultura Económica, México 2013.

36 Aristóteles, Tópicos, 101a34-b4, Gredos, Madrid 1982.

37 Ibíd., 163b28; Aristóteles, Retórica, 1403a18-19. 
evolución cultural, como apunta Michael Tomasello, se apoya en un «efecto trinquete», según el cual las nuevas generaciones retoman los artefactos previamente diseñados y son exploradas nuevas posibilidades prácticas a partir de estos ${ }^{38}$.

5. La neurorretórica atiende no sólo a los procesos mentales, sino también a los procesos emotivos ${ }^{39}$. Así, el mecanismo espejo, cuyas bases biológicas se constituyen por la activación de las neuronas espejo, sirve para comprender los efectos empáticos de simulación corporizada que se activan en los procesos de intercambio de emociones y sensaciones ${ }^{40}$. La observación de una percepción en otro individuo provoca la activación de las mismas áreas cerebrales que se activarían durante la experiencia perceptiva directa, e incluso estos mecanismos espejo permanecen también activos cuando nos imaginamos estar haciendo o percibiendo algo $\mathrm{o}^{41}$.

Al demostrar que cuando imaginamos una escena visual se activan las mismas áreas del cerebro implicadas en la participación efectiva en la misma escena, los investigadores sostienen que no sólo los límites entre el mundo real y el mundo imaginario son más difusos de lo que se pensaba, sino que las mismas relaciones interpersonales — pertenezcan a la realidad social o a los mundos posibles de la ficción- se distinguen de la implicación corpórea producida por las acciones, sentimientos y sensaciones de los otros. El auténtico descubrimiento consiste en haber demostrado cómo las percepciones corpóreas son incluso más potentes si se activan en relación con los estados de «inmersión» en mundos imaginarios que respecto a los estados de inmersión —-mucho más espurios, incontrolables e intermitentes - en el mundo real. La suspensión de nuestra posición en el mundo implica, más que una «suspensión de la incredulidad», una libre «simulación corporizada» en base a la cual actuamos y esperamos los acontecimientos como si fuéramos actores del mundo ficticio ${ }^{42}$.

6. La percepción del ecosistema en que vivimos y de los acontecimientos producidos en él exige que nuestro cerebro integre de manera coherente

38 Tomasello, M., Los orígenes culturales de la cognición humana, Amorrortu, Buenos Aires 2007.

39 Arenas-Dolz, F., «Neuro-retórica. ¿Vino viejo en odres nuevos?», en: Cortina, A. (ed.), Guía Comares de Neurofilosofía Práctica, Comares, Granada 2012, 125-145; Id., "¿Qué es la neurorretórica?», en: Daímon. Revista Internacional de Filosofía, 58, 2013, 69-80; Id., "Neurofilosofía, naturalismo y juicio moral», en: CARbonell, C. y Flamarique, L. (eds.), De simios, cyborgs y dioses: La naturalización del hombre a debate, Biblioteca Nueva, Madrid 2016, 207-223.

40 Gallese, V. y Wojcienowski, H. C., «How stories make us feel: Toward an embodied narratology», en: Californian Italian Studies, 2/1, 2011, 24.

${ }^{41}$ Gallese, V., "The "shared manifold" hypothesis: From mirror neurons to empathy", en: Journal of Consciousness Studies, 8/5-7, 2001, 41-47; GALLESE, V., «The manifold nature of interpersonal relations: The quest for a common mechanism», en: Philosophical Transactions of the Royal Society of London, B, 2003, 527.

42 Gallese, V., «Il corpo teatrale: mimetismo, neuroni specchio, simulazione incarnata», en: Culture Teatrali, 16, 2008, 28-30. 
informaciones provenientes de distintos canales sensoriales. La investigación neurocientífica ha contribuido a desmantelar una concepción modular de la percepción, demostrando que los sentidos no operan de manera separada, sino que los mecanismos perceptivos se basan en su interacción ${ }^{43}$.

Las investigaciones realizadas en el ámbito neurocientífico han estudiado la percepción intermodal (también llamada intersensorial o multimodal), que consiste en la capacidad de recibir información simultánea a través de distintos canales sensoriales de un mismo suceso y de una forma integrada. Es la percepción unitaria o unificada de objetos o eventos a partir de estímulos simultáneos disponibles a través de más de un canal sensorial. Es decir, que el individuo es capaz de establecer una relación entre dos tipos de información, por ejemplo, reconociendo el vínculo entre una voz y la imagen de la persona que habla como un único acontecimiento.

Precisamente estos estudios neurocientíficos sobre la percepción han suscitado en la actualidad un renovado interés por la sinestesia. La sinestesia es el procedimiento retórico que consiste en mezclar sensaciones percibidas por órganos sensoriales distintos, como sucede por ejemplo cuando describimos una experiencia gustativa valiéndonos de un código léxico relativo al tacto. Pero además de ser un mecanismo retórico basado en la transferencia de significado entre dos o más sistemas sensoriales, la sinestesia representa también un fenómeno de tipo neurofisiológico, como cuando un sonido determina impresiones visuales en los casos de la audición coloreada (el do es rojo, el re verde, etc. ${ }^{44}$.

El extraordinario interés por la sinestesia ha sido promovido por la ciencia cognitiva corporizada al reconocer como fundamental la relación que la cognición tiene con la corporeidad, la emoción, la afectividad y la acción ${ }^{45}$. Se trata de un fenómeno que ha suscitado interés en el ámbito literario, filosófico y psicológico desde finales del siglo xIx. Por ejemplo, Francis Galton en sus Inquires into human faculty and its development nos proporciona los primeros datos cuantitativos sobre la sinestesia ${ }^{46}$. Este mismo año se publicó por primera vez el soneto Vocales de Arthur Rimbaud ${ }^{47}$, donde el poeta francés - que buscaba ilustrar la teoría de las correspondencias expuesta en 1857 en Las flores del mal por Charles Baudelaire ${ }^{48}$, quien a su vez se inspira en Emanuel Swedenborg - le da un color a cada una de las vocales: 'a' negra, 'e' blanca, 'i' roja, 'u' verde y 'o' azul (nótese que la 'o' aparece al final, en vez de la 'u').

43 Calvert, G. A.; Spence, C. y Stein, B. E., The handbook of multisensory processes, MIT Press, Cambridge 2004.

44 Mattingley, J. B., Cognitive neuroscience perspective on synaesthesia, Masson, New York 2006.

45 Varela, F. J.; Thompson, E. y Rosch, E., o. $c$.

46 GaLton, F., Inquires into human faculty and its development, Macmillan, London 1883.

47 Rimbaud, A., Poesías completas, Cátedra, Madrid 2005.

48 Baudelaire, C., Las flores del mal, Cátedra, Madrid 1997. 
Durante cierto tiempo, la explicación más común de la sinestesia había sido en términos de asociaciones aprendidas, posiblemente durante la infancia. Vilayanur Ramachandran y Edward M. Hubbart han propuesto un modelo de interconexión local que puede explicar por qué una persona puede experimentar varias sinestesias ${ }^{49}$. El hecho de que se observe con frecuencia en miembros de la misma familia ha llevado a algunos investigadores a sugerir una base genética, aunque los datos no son concluyentes ${ }^{50}$. Una prueba muy utilizada para mostrar que las experiencias sinestésicas son genuinas y automáticas es el test Stroop, en el cual nombrar un color es más lento cuando el color auténtico de un estímulo está en conflicto con el color generado por la sinestesia ${ }^{51}$.

7. A diferencia de lo que sucede en otras especies, en los seres humanos no existe un dominio sensorial prevalente, sino que se da una constante interrelación entre los distintos sistemas perceptivos. El cerebro está formado por diferentes áreas dispuestas de forma regular, cada una de las cuales tiene asignada la elaboración de sensaciones determinadas, pero es la presencia de estructuras sinápticas especializadas en la comunicación entre las neuronas la que favorece la conexión intermodal entre estas áreas.

$\mathrm{Al}$ respecto son fundamentales las investigaciones coordinadas por Giacomo Rizzolatti, que ha mostrado la existencia de las neuronas espejo, responsables tanto del ámbito perceptivo como del motor. Esta clase de neuronas visivo-motoras ha sido identificada en algunas áreas cerebrales, primero en primates y luego en humanos. Las neuronas se activan — «descargan», como dicen los neurocientíficos, es decir, producen una cantidad mayor de impulsos eléctricos- cuando se realiza alguna tarea y además cuando se observa esa misma tarea específica desarrollada por otro. El sistema espejo se activa también en los casos en que no es posible observar plenamente la interacción entre la mano de quien realiza el experimento y el objeto, sino sólo inferirla ${ }^{52}$.

Estos descubrimientos han mostrado que observar las acciones de otro significa registrarlas en nuestro sistema visual para verterlas después, mapearlas, en nuestras representaciones motoras: las comprendemos en la medida en que hemos tenido una experiencia directa, aunque simulada.

El sistema espejo es importante para comprender las bases neurofisiológicas de las acciones de otras personas (empatía) y para aprender nuevas

49 Ramachandran, V. S. y Hubbard, E. M., «Synaesthesia: A window into perception, thought and language», en: Journal of Consciousness Studies, 8/12, 2001, 3-34.

50 Baron-Cohen, S.; Burt, L.; Smith-Laittan, F.; Harrison, J. y Bolton, P., «Synaesthesia: Prevalence and familiarity», en: Perception, 25/9, 1996, 1073-1080.

51 Odgaard, E. C.; Flowers, J. H. y Bradman, H. L., «An investigation of the cognitive and perceptual dynamics of a colour-digit synaesthete», en: Perception, 28, 1999, 651-664.

52 Rizzolatti, G. y Vozza, L., Nella mente degli altri. Neuroni specchio e comportamento sociale, Zanichelli, B. 2006; Rizzolatti, G. y Sinigaglia, C., Mirrors in the brain: How our minds share actions and emotions, Oxford University Press, Oxford 2008. 
habilidades por imitación (mímesis) ${ }^{53}$. Para la neurorretórica el sistema espejo puede compararse a una especie de proceso metafórico que parte de un repertorio motor conocido para describir algo desconocido, esto es, las acciones de los otros ${ }^{54}$. Comprendemos los gestos y comportamientos de los otros repitiéndolos virtualmente, en un proceso mimético anclado en el cuerpo. Del mismo modo que la credibilidad del orador es un factor determinante de la persuasión y se obtiene al identificarse con las emociones del auditorio y observar atentamente sus actitudes, especialmente las no verbales, desde la infancia aprendemos mediante simulaciones, identificaciones y actos empáticos. Es un hecho que el juego simbólico o de imitación en la infancia, especialmente entre los 3 y 5 años, nos ayuda a usar mejor el lenguaje y a entrar en empatía con el otro.

Sin embargo, la interacción con los otros no puede reducirse a los procesos de imitación y especulares, por lo que la sociabilidad humana es inseparable del sentido de un "mundo compartido" hecho de valores compartidos $^{55}$. Este sentido surge de la interacción con los otros a través de complejos sistemas de reciprocidad. Como han señalado Peter L. Berger y Thomas Luckmann: «En la vida cotidiana el conocimiento aparece distribuido socialmente, vale decir que diferentes individuos y tipos de individuos lo poseen en grados diferentes ${ }^{56}$. La intersubjetividad humana se desarrolla adecuadamente gracias a los intercambios sociales recíprocos, que no son sólo imitativos, sino selectivos e intencionales, y contribuyen al aprendizaje cultural en general.

8. Los resultados de las investigaciones muestran que los sinestésicos poseen quizá una mayor capacidad para establecer asociaciones inéditas, elemento que podría representar una fuente de inspiración para quienes se dedican a prácticas artísticas, aunque no sea posible afirmar que sinestesia y creatividad tengan bases genéticas o neuronales comunes ${ }^{57}$. Más importante ha resultado el concepto de esquema de imagen (image schema) introducido por primera vez en 1987 por el filósofo estadounidense Mark Johnson y convertido hoy en la clave para encontrar en el cuerpo los orígenes de los procesos de significación y del pensamiento ${ }^{58}$. Según Johnson un esquema

53 Iacoвоni, M., Mirroring people: The science of empathy and how we connect with others, Picador, New York 2009.

54 Fogassi, L., «La metafora nel cervello: il punto di vista neurofisiologico», en: Lorusso, A. M. (ed.), Metafora e conoscenza. Da Aristotele al cognitivismo contemporaneo, Bompiani, M. 2005, 349-361.

${ }_{55}$ SchüTz, A., La construcción significativa del mundo social. Introducción a la sociología comprensiva, Paidós, Barcelona 1993.

56 Berger, P. L. y Luckmann, T., La construcción social de la realidad, Amorrortu, Buenos Aires 2008, 63.

57 Gallese, V. y Freedberg, D., «Movimento, emozione, empatia. I fenomeni che si producono a livello corporeo osservando le opere d'arte», en: Prometeo, 103, 2008, 52-61.

58 Hampe, B. y Grady, J. E. (eds.), From perception to meaning: Image schemas in cognitive linguistics, Mouton de Gruyter, Berlin-New York 2005. 
de imagen es un «patrón dinámico que funciona a la manera de la estructura abstracta de una imagen y, por tanto, conecta una extensa variedad de experiencias distintas que ponen de manifiesto la misma estructura recurrente» ${ }^{59}$. Se trata de estructuras conceptuales directamente significantes que emergen de los movimientos del cuerpo en el espacio, de las interacciones perceptivas y del modo en que manipulamos los objetos, es decir, de comportamientos perceptivos altamente esquemáticos que capturan la esencia de la experiencia sensomotora, integrando la información proveniente de modalidades diferentes y proporcionando al cuerpo un anclaje en el sistema conceptual. Ejemplos de esquemas de imagen son nociones como fuente-trayectoria-meta, continente-contenido, centro-periferia, enlace, fuerza, equilibrio, etc. y es sólo gracias a ellos que logramos traducir la sensomotricidad en un régimen de abstracción.

Configuraciones primigenias, que mapean la experiencia corporal cotidiana y al mismo tiempo tienen una función esencial en la estructuración de nuestro mundo mental, los esquemas de imagen son importantes para la neurorretórica porque constituyen de alguna manera sus materiales de construcción. Las cinco partes en que se articulaba la retórica clásica inventio, dispositio, elocutio, memoria, actio- convergen en la noción de image schema precisamente porque son capaces de concebir las interacciones entre organismo y ambiente en términos a la vez físicos y mentales ${ }^{60}$.

Si para la retórica clásica persuadir significaba situarse en un contexto físico determinado para emitir desde allí el mensaje más persuasivo y convincente, también en la perspectiva de Johnson la mente humana aparece como corporizada, y la significación, el pensamiento, las expresiones simbólicas están arraigados en los modelos perceptivos y en los movimientos corporales. A diferencia de los conceptos más abstractos de esquema y guión, la noción de esquema de imagen subraya la naturaleza corporizada de los procesos cognitivos y postula la existencia de modelos recurrentes de naturaleza sensomotora, cuya función sería hacer significativa la experiencia. En la arquitectura neuronal existen redes encargadas exclusivamente del movimiento, la extensión, la dirección de las células, y mediante el uso de términos como «arriba», «abajo», «fuera», «a través», etc. nos ayudan a referirnos correctamente a las relaciones espaciales y a los movimientos, así como a visualizar objetos estáticos o dinámicos: sabemos que las áreas del cerebro no son sensibles a un solo tipo de estímulo sino que están estrechamente interconectadas, por lo que no sólo una misma información puede utilizarse para distintos propósitos de diferentes áreas del cerebro, sino que es posible que el mismo esquema de imagen se aplique a

59 Johnson, M., El cuerpo en la mente. Fundamentos corporales del significado, la imaginación y la razón, Debate, Madrid 1991, 50.

60 Langacker, R. W., Grammar and conceptualization, Mouton de Gruyter, Berlin 1999. 
experiencias diferentes, o viceversa que a una misma experiencia se apliquen más esquemas de imagen ${ }^{61}$.

Pese a depender de las percepciones corporales, de las acciones y de los sentimientos, los esquemas de imagen corresponden sólo a los organismos dotados de una particular estructura cerebral: por ejemplo un conocido esquema de imagen es el de «horizonte», relacionado directamente con la simetría bilateral de nuestro cuerpo gracias a la que tenemos conocimiento de la derecha y la izquierda, de delante y detrás, de lo próximo y lo lejano. Ligados a determinadas situaciones de la experiencia corporal e impregnados de percepciones visuales, auditivas, táctiles, olfativas y cinestésicas así como a sensaciones internas como el hambre y el dolor, los esquemas de imagen no son sólo representaciones esquemáticas de la experiencia física sino el anclaje de la cognición, los cimientos del sistema de autoorganización humana que se recrean y se vuelven a experimentar durante la actividad cognitiva y perceptiva: los datos propioceptivos que provienen de las terminaciones nerviosas presentes en los músculos y en la piel nos proporcionan de hecho informaciones sobre el movimiento, la posición y la postura, gracias a los cuales estamos en condiciones de movernos ${ }^{62}$.

La sinestesia ha sido uno de los ámbitos privilegiados de aplicación del concepto de esquema de imagen, porque si la mayor parte de las investigaciones se han concentrado en la dimensión visual y cinestésica de la organización de la experiencia, atribuyendo a la vista un papel predominante en la descodificación de datos perceptivos, otros autores han demostrado que los esquemas de imagen no se refieren exclusivamente a propiedades visuales, sino también a datos de tipo táctil, y en concreto a la percepción háptica. Para demostrar la naturaleza intermodal de los esquemas de imagen, Yanna Popova ha estudiado el esquema de imagen «ajuste escalar» - que se refiere a la construcción de un evento en varios grados de abstracción o esquematización-, conjeturando que está enraizado en el tacto, y el fenómeno de la sinestesia le sirve precisamente para verificar esta hipótesis ${ }^{63}$.

El descubrimiento de la importancia del tacto es un aspecto crucial para la neurorretórica, pues nos permite comprender la metáfora desde una perspectiva que no tenga en la visión su punto de referencia exclusivo. Numerosos estudios preparados por cognitivistas y neurocientíficos han

61 Dodge, E. y Lakoff, G., «Image schemas: From linguistic analysis to neural grounding», en: HAMPe, B. y Grady, J. E. (eds.), From perception to meaning: Image schemas in cognitive linguistics, Mouton de Gruyter, Berlin-New York 2005, 57-91.

62 Damasio, A. R., o. c.; Grady, J. E., «Image schemas and perception: Refining a definition», en: HAMPe, B. y Grady, J. E. (eds.), From perception to meaning: Image schemas in cognitive linguistics, Mouton de Gruyter, Berlin-New York 2005, 36-55.

63 Popova, Y., "Image schemas and verbal synaesthesia», en: Hampe, B. y Grady, J. E. (eds.), From perception to meaning: Image schemas in cognitive linguistics, Mouton de Gruyter, Berlin-New York 2005, 395-421. 
mostrado que los esquemas de imagen equivalen a tropos, en particular a metáforas ${ }^{64}$. La experiencia táctil no es secundaria en absoluto. Provocada por variaciones continuas en el contacto entre el cuerpo y un agente externo, se desarrolla de modo secuencial en el tiempo, ya que tales variaciones constituyen en un primer momento detalles aislados que sólo después son recompuestos en una unidad coherente de significado. Además, el tacto contribuye de modo determinante a la percepción del espacio, como muestra el grado de comprensión de la organización espacial en las personas ciegas de nacimiento, muy parecido al de las videntes, o el hecho que en niños de dos meses el reconocimiento de la forma y el tamaño de los objetos mediante la mera manipulación es el mismo que se tendría con la vista. Con el gusto y el olfato, el tacto participa en la constitución de informaciones complejas como las relativas al sabor de los alimentos, mientras que junto con el oído es responsable de la percepción de las ondas acústicas, y con la vista contribuye a reconocer la forma y naturaleza de los objetos (estereognosis).

\section{A MOdo DE CONCLUSIÓN}

Ya para Aristóteles el discurso se construía en torno a aspectos lógicos (logos), pero también éticos (ethos) y afectivos (pathos): «De entre los medios de credibilidad, los que pueden obtenerse mediante el discurso son de tres especies: unos residen en el carácter del que habla, otros en predisponer al oyente de alguna manera y, los últimos, en el discurso mismo, merced a lo que este demuestra o parece demostrar» ${ }^{65}$. La retórica aristotélica mostraba así el factum de la «unidad vital», anterior a toda separación entre lo cognitivo, lo volitivo y lo afectivo. Tras este modelo se encontraba la concepción aristotélica de la actividad humana, siendo la retórica la encargada de sistematizar los procedimientos que hacen comunicable la realidad intelectual y emotiva del hombre.

Para los defensores de las teorías ambientalistas la cognición se inserta en situaciones en las cuales hemos de responder a las novedades imprevistas del ambiente. La inteligencia humana comprende un ámbito de actuaciones que no se reduce a las formas tradicionales de resolución de problemas mediante el razonamiento, la planificación o la reflexión. El mundo mismo se convierte en el modelo sobre el cual se ejecutan los procesos de razonamiento, de generación de planes y de reestructuración de las competencias cognitivas. Los defensores de la cognición situada, por ejemplo, creen que la mejor alternativa es pensar que el agente está situado, incrustado, inmerso en el mundo que la

64 GEARY, J., I is an other: The secret life of metaphor and how it shapes the way we see the world, Harper, New York 2012.

65 ARISTóteles, Retórica, 1356a2-4. 
organización de las tareas exige una respuesta a variaciones en tiempo real, es decir, una mayor sensibilidad a las condiciones de cada situación.

En la actualidad, la neurorretórica, surgida de la convergencia entre la retórica y las ciencias neurocognitivas, ha mostrado cómo se encuentran y compenetran las funciones cognitivas y emocionales, descubriendo nuevos ámbitos de aplicación de la retórica. Desde esta perspectiva, la retórica, más que arte de la persuasión, teoría de la argumentación o teoría literaria, es principalmente una ciencia acerca de cómo estructuramos nuestro conocimiento a través del lenguaje, acerca de cómo el ser humano construye su mundo a través del logos. Los tropos y figura retóricas no son meros instrumentos comunicativos y literarios, sino redes conceptuales que estructuran el pensamiento humano.

Si las neurociencias han intentado traducir en términos de operaciones cognitivas y configuraciones neuronales conceptos retóricos conocidos desde antiguo es precisamente por la importancia de estos aspectos en el juicio moral. El cerebro dota al ser humano de herramientas para interactuar con el medio y convertirlo en un mundo con sentido. En particular, las investigaciones sobre la sinestesia y la plasticidad cerebral han llevado a los lingüistas cognitivos a revisar las teorías sobre la evolución del lenguaje; el descubrimiento del sistema de neuronas espejo ofrece las bases biológicas de las cuales surge el significado y la comprensión. Todos estos estudios han dado un renovado impulso a la retórica promoviendo una visión no reductiva de la misma.

\section{Bibliografía}

Arenas-Dolz, F., «Neuro-retórica. ¿Vino viejo en odres nuevos?», en: Cortina, A. (ed.), Guía Comares de Neurofilosofía Práctica, Comares, Granada 2012, 125-145.

Arenas-Dolz, F., «¿Qué es la neurorretórica?», en: Daímon. Revista Internacional de Filosofía, 58, 2013, 69-80.

Arenas-Dolz, F., «Neurofilosofía, naturalismo y juicio moral», en: CArbonell, C. y FlaMARIQUE, L. (eds.), De simios, cyborgs y dioses: La naturalización del hombre a debate, Biblioteca Nueva, Madrid 2016, 207-223.

ARIstóteles, Tópicos, Gredos, Madrid 1982.

Aristóteles, Retórica, Gredos, Madrid 1992.

BARABÁSI, A.-L., Linked: How everything is connected to everything else and what it means for business, science, and everyday life, Perseus Publishing, Cambridge 2002.

Baron-Cohen, S.; Burt, L.; Smith-Laittan, F.; Harrison, J. y Bolton, P., "Synaesthesia: Prevalence and familiarity», en: Perception, 25/9, 1996, 1073-1080.

Baudelaire, C., Las flores del mal, Cátedra, Madrid 1997.

Berger, P. L. y Luckmann, T., La construcción social de la realidad, Amorrortu, Buenos Aires 2008.

Bеuснот, М., La retórica como pragmática y hermenéutica, Anthropos, Barcelona 1998.

BRоокs, R. A., «Intelligence without representation», en: Artificial Intelligence, 47, 1991, 139-159.

Bruner, J. S., La fábrica de historias. Derecho, literatura, vida, Fondo de Cultura Económica, México 2013. 
Calvert, G. A.; Spence, C. y Stein, B. E., The handbook of multisensory processes, MIT Press, Cambridge 2004.

CLARK, A., Being there: Putting brain, body, and world together, MIT Press, Cambridge 1997.

CLARK, A., Supersizing the mind: Embodiment, action, and cognitive extension, Oxford University Press, New York 2008.

Clark, A. y Chalmers, D. J., «The extended mind», en: Analysis, 58, 1998, 7-19.

Christakis, N. A. y Fowler, J. H., Conectados. El sorprendente poder de las redes sociales y cómo nos afectan, Taurus, Madrid 2010.

Conill, J., «Retórica y vida humana», en: Arenas-Dolz, F. (ed.), Retórica y democracia. Perspectivas críticas sobre el estado de la investigación, Institució Alfons el Magnànim, Valencia 2012, 323-339.

Coronel Ramos, M. A. y Giménez Moreno, R., «Cognición y retórica», en: Arbor, 177.697, 2004, 41-58.

Damasio, A. R., El error de Descartes. La emoción, la razón y el cerebro humano, Crítica, Barcelona 2001.

Dehaene, S., Les neurones de la lecture, Odile, Paris 2007.

Dehaene, S., El cerebro lector, Siglo xxi, Buenos Aires 2014.

Dodge, E. y LaKoff, G., «Image schemas: From linguistic analysis to neural grounding», en: Hampe, B. y Grady, J. E. (eds.), From perception to meaning: Image schemas in cognitive linguistics, Mouton de Gruyter, Berlin-New York 2005, 57-91.

Downes, S., "An introduction to connective knowledge», en: Hug, T. (ed.), Media, knowledge \& education: Exploring new spaces, relations and dynamics in digital media ecologies [en línea], Innsbruck University Press, Innsbruck 2008, 77-102 [consultado 30 mayo 2016]. Disponible en Internet: <http://www.oapen.org/search?identifier=449459>.

FogASSI, L., «La metafora nel cervello: il punto di vista neurofisiologico», en: Lorusso, A. M. (ed.), Metafora e conoscenza. Da Aristotele al cognitivismo contemporaneo, Bompiani, Milano 2005, 349-361.

Gallese, V., «The "shared manifold” hypothesis: From mirror neurons to empathy», en: Journal of Consciousness Studies, 8/5-7, 2001, 33-50.

GaLlese, V., "The manifold nature of interpersonal relations: The quest for a common mechanism», en: Philosophical Transactions of the Royal Society of London, B, 2003, 358, 517-528.

GALLESE, V., «Il corpo teatrale: mimetismo, neuroni specchio, simulazione incarnata», en: Culture Teatrali, 16, 2008, 13-38.

Gallese, V., «Mirror neurons and the social nature of language: The neural exploitation hypothesis», en: Social Neuroscience, 3/3-4, 2008, 317-333.

Gallese, V. y Freedberg, D., «Movimento, emozione, empatia. I fenomeni che si producono a livello corporeo osservando le opere d'arte», en: Prometeo, 103, 2008, 52-61.

Gallese, V.; Migone, P. y Eagle, M. N., «La simulazione incarnata: i neuroni specchio, le basi neurofisiologiche dell'intersoggettività ed alcune implicazioni per la psicoanalisi», en: Psicoterapia e Scienze Umane, 12.3, 2006, 543-580.

Gallese, V. y Wojciehowski, H. C., «How stories make us feel: Toward an embodied narratology», en: Californian Italian Studies, 2/1, 2011, 3-37.

Galton, F., Inquires into human faculty and its development, Macmillan, London 1883.

GEARY, J., I is an other: The secret life of metaphor and how it shapes the way we see the world, Harper, New York 2012.

Goody, J., La lógica de la escritura y la organización de la sociedad, Alianza, Madrid 1990. 
GRADY, J. E., «Image schemas and perception: Refining a definition», en: Hampe, B. y GRADY, J. E. (eds.), From perception to meaning: Image schemas in cognitive linguistics, Mouton de Gruyter, Berlin-New York 2005, 36-55.

Griffiths, P. E. y Scarantino, A., "Emotions in the wild», en: Robbins, P. y Aydede, M. (eds.), The Cambridge handbook of situated cognition, Cambridge University Press, Cambridge 2009.

Hampe, B. y Grady, J. E. (eds.), From perception to meaning: Image schemas in cognitive linguistics, Mouton de Gruyter, Berlin-New York 2005.

Havelock, E. A., Prefacio a Platón, Visor, Madrid 1994.

Herman, D., Story logic: Problems and possibilities of narrative, University of Nebraska Press, Lincoln 2002.

Herman, D. (ed.), Narrative theory and the cognitive sciences, Center for the Study of Language and Information, Stanford 2003.

Hutchins, E., Cognition in the wild, MIT Press, Cambridge 1995.

Iacoвoni, M., Mirroring people: The science of empathy and how we connect with others, Picador, New York 2009.

JACK, J. y Appelbaum, L. G., "This is your brain on rhetoric': Research directions for neurorhetorics», en: Rhetoric Society Quarterly, 40/5, 2010, 411-437.

Johnson, M., El cuerpo en la mente. Fundamentos corporales del significado, la imaginación y la razón, Debate, Madrid 1991.

Lakoff, G. y Johnson, M., Metáforas de la vida cotidiana, Cátedra, Madrid 2007.

Langacker, R. W., Grammar and conceptualization, Mouton de Gruyter, Berlin 1999.

LÉvy, P., Inteligencia colectiva. Por una antropología del ciberespacio, Organización Panamericana de la Salud, Washington 2004.

Mattingley, J. B., Cognitive neuroscience perspective on synaesthesia, Masson, New York 2006.

Maturana, H., Erkennen: Die Organisation und Verkörperung von Wirklichkeit, Friedrich Vieweg und Sohn, Braunschweig/Wiesbaden 1982.

Maturana, H. y Varela, F. J., El árbol del conocimiento. Las bases biológicas del entendimiento humano, Debate, Madrid 1990.

Mesquita, B., «Emoting: A contextualized process», en: Mesouita, B.; Barrett, L. F. y Sмітн, E. R. (eds.), The mind in context, Guilford Press, New York 2010, 83-104.

Minsky, M. L., La sociedad de la mente. La inteligencia humana a la luz de la inteligencia artificial, Galápago, Buenos Aires 1986.

Newell, A. y Simon., H. A., "Computer science as empirical inquiry», en: Communications of the ACM, 19, 1976, 113-126.

Odgatrd, E. C.; Flowers, J. H. y Bradman, H. L., «An investigation of the cognitive and perceptual dynamics of a colour-digit synaesthete», en: Perception, 28, 1999, 651-664.

Ong, W. J., Oralidad y escritura. Tecnologías de la palabra, Fondo de Cultura Económica, México 1987.

Perelman, C. y Olbrechts-Tyteca, L., Tratado de la argumentación. La nueva retórica, Gredos, Madrid 1989.

Platón, Gorgias, Gredos, Madrid 1992.

Popova, Y., «Image schemas and verbal synaesthesia», en: HAMPE, B. y Grady, J. E. (eds.), From perception to meaning: Image schemas in cognitive linguistics, Mouton de Gruyter, Berlin-New York 2005, 395-421.

RAMACHANDRAN, V. S. y Hubbard, E. M., «Synaesthesia: A window into perception, thought and language», en: Journal of Consciousness Studies, 8/12, 2001, 3-34. 
Ramírez, J. L., "Seguidores o continuadores de la retórica clásica», en: Arenas-Dolz, F. (ed.), Retórica y democracia. Perspectivas críticas sobre el estado de la investigación, Institució Alfons el Magnànim, Valencia 2012, 341-366.

Rimbaud, A., Poesías completas, Cátedra, Madrid 2005.

Rizzolatti, G. y Sinigaglia, C., Mirrors in the brain: How our minds share actions and emotions, Oxford University Press, Oxford 2008.

Rizzolatti, G. y Vozza, L., Nella mente degli altri. Neuroni specchio e comportamento sociale, Zanichelli, Bologna 2006.

Rumelhart, D. E.; McClelland, J. L. y PDP Research Group (eds.), Parallel distributed processing, MIT Press, Cambridge 1986.

Salomon, G. (ed.), Cogniciones distribuidas. Consideraciones psicológicas y educativas, Amorrortu, Buenos Aires 2001.

Schank, R. C. y Abelson, R. P., Guiones, planes, metas y entendimiento. Un estudio de las estructuras del conocimiento humano, Paidós, Barcelona 1987.

Schüтz, A., La construcción significativa del mundo social. Introducción a la sociología comprensiva, Paidós, Barcelona 1993.

Siemens, G., Conociendo el conocimiento [en línea], Nodos Ele, Madrid 2010 [consultado 30 mayo 2016]. Disponible en Internet: <http://www.nodosele.com/editorial>.

Stamenov, M. I. y Gallese, V., Mirror neurons and the evolution of brain and language, John Benjamins, Amsterdam 2002.

Suchman, L., Plans and situated actions: The problem of human-machine communication, Cambridge University Press, New York 1987.

Suchman, L., «Representing practice in cognitive sciences», en: Human Studies, 11/2-3, $1988,305-325$.

Tomasello, M., Los orígenes culturales de la cognición humana, Amorrortu, Buenos Aires 2007.

VAN GELDER, T., «What might cognition be, if not computation?», en: Journal of Philosophy, 91, 1995, 345-381.

VAN GELDER, T., "The dynamical hypothesis in cognitive science», en: Behavioral and Brain Sciences, 21, 1998, 615-628.

Varela, F. J.; Thompson, E. y Rosch, E., De cuerpo presente. Las ciencias cognitivas y la experiencia humana, Gedisa, Barcelona 1992.

Vygotski, L. S., El desarrollo de los procesos psicológicos superiores, Crítica, Barcelona 2000.

Wolf, M., Cómo aprendemos a leer. Historia y ciencia del cerebro y la lectura, Ediciones B, Barcelona 2008.

Zunshine, L., Why we read fiction? Theory of mind and the novel, Ohio State University Press, Columbus 2006.

Departament de Filosofia

Francisco Arenas-Dolz

Facultat de Filosofia i Ciències de l'Educació

Universitat de València

Av. Blasco Ibáñez 30

E-46010 Valencia (España)

francisco.arenas@uv.es

[Artículo aprobado para publicación en octubre de 2016]. 